\title{
Single-Molecule Device Prototypes for Protein-Based Nanoelectronics: Negative Differential Resistance and Current Rectification in Oligopeptides
}

\author{
David M. Cardamone and George Kirczenow \\ Physics Department, Simon Fraser University, Burnaby, BC V5A 1S6, Canada
}

\begin{abstract}
We investigate electrical conduction through individual oligopeptide molecules thiol-bonded between gold nanocontacts using $a b$ initio and semi-empirical techniques. Our theory explains for the first time these molecules' experimentally observed current-voltage characteristics, including both the magnitude and rectification of the current, and uses no adjustable parameters. We identify the mechanism of the observed current rectification, and predict that it will result in negative differential resistance at moderate biases. Our findings open the way to the realization of protein-based nanoelectronic devices.
\end{abstract}

\section{INTRODUCTION}

Living cells survive and function through their unique ability to manufacture a vast catalog of specific molecules repeatably and reliably, a capacity long recognized to hold promise for the design of nanoelectronic devices. $\frac{1}{n}$ Whereas much experimental and theoretical work has been devoted to understanding the electron transport properties of single nucleic acid molecules, relatively little has focused on the same qualities of other biomolecules. In particular, over the course of its life a cell produces far more polypeptide chains than DNA sequences, while amino acids, the building blocks of proteins, display five times more diversity than DNA bases. As such, the single-molecule electron transport characteristics of these protein fragments, and of entire proteins, is a field with great potential, which is only beginning to be explored.

Recently, experimentalists have succeeded in addressing the effects of primary protein structure, i.e. specific amino acid sequences, on conductance $\stackrel{2.3}{=}$ These experiments, applying the STM-break junction (STMBJ) technique 4.5.6 $^{-6}$ to oligopeptide molecules, present an exciting new approach to biologically-based nanoelectronic devices: They offer a broad class of well defined experimental systems operating at length scales on which coherent quantum effects are dominant. Reference 3 , in particular, demonstrated this promise with non-equilibrium results evincing a striking current rectification.

The purpose of this article is to present the first theoretical treatment of electron transport through these systems. We explain the phenomena observed in these experiments, in particular the current rectification, by use of Landauer conductance theory,$\underline{7}$ and demonstrate good quantitative agreement with the experimental data. We show that the rectification is the result of resonance between gold-molecule interfacial states partially localized on either side of the molecule (see Fig. 1); these states are detuned from each other in equilibrium due to the oligopeptides' intrinsic asymmetry. Moreover, we predict that, at moderately higher voltages, a related mechanism will generate negative differential resistance (NDR), i.e., a decrease in current with increased bias voltage; this phenomenon has important device applications, including low-noise amplification,,$\stackrel{9}{,}$ high frequency oscillators,$\underline{10}$

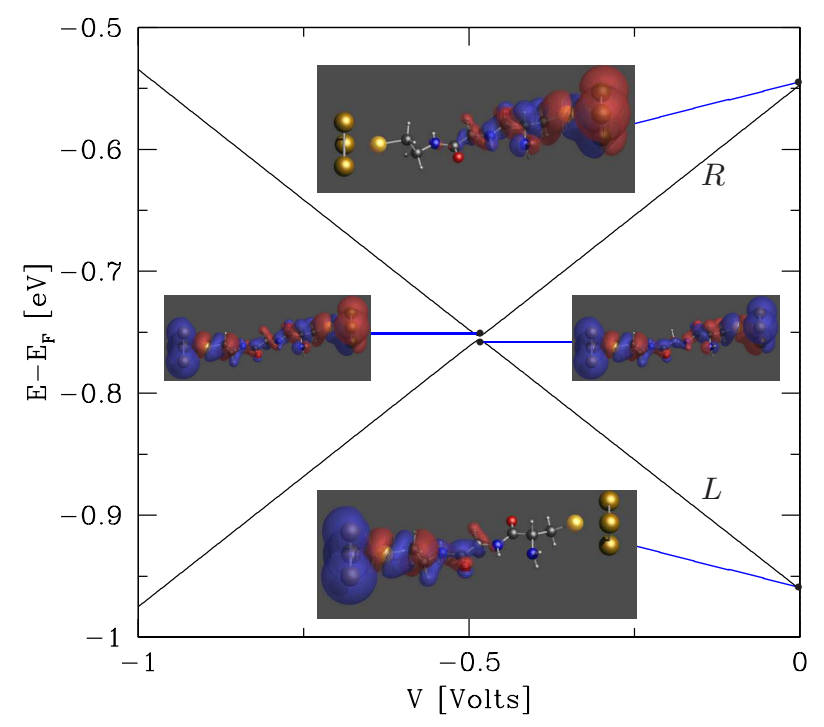

FIG. 1: (Color online) Anticrossing of generalized eigenstates $R$ and $L$ of the simplified extended molecule discussed in the text for cysteamine-Gly-Cys bonded to two hollow sites in the $\mathrm{Au}(111)$ leads. In the full system, the anticrossing's abruptness is tempered by broadening from the leads. The insets show the states at specific bias voltages. ${ }^{-}$

analog-to-digital conversion,,$\frac{11}{1}$ and digital logic, 12 Thus, our findings open the way to the realization of practical oligopeptide, and ultimately protein-based, nanoelectronic devices.

We organize the paper as follows. Section $\Pi$ presents the theoretical formalism we use to model charge transport through metal-oligopeptide-metal junctions. In Section III we discuss our results for these systems, including determination of the experimental geometry, explanation of their rectifying properties, and prediction of NDR. Section IV summarizes our main conclusions. 


\section{MODEL}

To determine the molecular geometry in the presence of the gold leads, we apply a series of density functional theory $a b$ initio calculations to relax the molecule between two fixed gold atoms ${ }^{13}$ At each step, the distance between the two atoms is increased slightly, and the geometry relaxed again, to simulate the stretching the molecule undergoes in an STMBJ experiment. For calculations reported here, the molecules have been stretched to their greatest length before breakage occurs, although we find that less stretched molecules have essentially the same conductance features and order of magnitude. In the case of hollow binding sites, we replace the gold atom with the three nearest-neighbor gold atoms, and relax the molecule one final time.

To determine conductance and current, we first partition the metal-oligopeptide-metal system into left and right macroscopic gold electrodes, and an extended molecule containing the central organic molecule as well as 50-100 atoms of each $\mathrm{Au}(111)$ contact. 14 The Hamiltonian of the full system is therefore the sum of two terms:

$$
H=H_{m o l}+H_{\text {leads }} .
$$

$H_{\text {mol }}$ describes the isolated extended molecule, and $H_{\text {leads }}$ does the same for the macroscopic electrodes, as well as containing the quantum mechanical coupling between them and the extended molecule.

We employ a semi-empirical model for $H_{m o l}$; such models are known to be highly effective in predicting, reproducing, and explaining experimental results for metal-organic molecule-metal junctions (e.g., Ref. 15, Ref. 16 and the results of Refs. 17 and 6, Ref. 18). The Hamiltonian of the full extended molecule is given by extended Hückel theory, 19,20,21,22,23,24

$H_{\text {leads }}$ provides both decoherent broadening and elastic scattering to the discrete states of the extended molecule. To model this, we assign semi-infinite, one-dimensional $\operatorname{leads}^{25}$ to each gold orbital not immediately adjacent to the oligopeptide; their number is equal to the coordination number of gold minus the number of nearestneighbor atoms included in the extended molecule. We take the intersite hopping within each lead, as well as the hopping between lead and orbital, to be $5 \mathrm{eV}$. These choices are made to quantitatively reproduce the quantized conductance $\frac{26,27}{2}$ of a gold quantum point contact, and are not fitted to any molecular electronics experiment.

The open system described by $H$ requires an infinite Fock space. To solve the full molecule-plus-leads system, then, we make use of Dyson's Equation, which gives the retarded Green function

$$
G(E)=\left[G_{m o l}^{-1}(E)-\Sigma(E)\right]^{-1} .
$$

Here

$$
G_{m o l}(E)=\frac{1}{S E-H_{m o l}+i 0^{+}}
$$

is the Green function for the extended molecule only, 28 and the retarded self-energy $\Sigma(E)$ is given by Ref. 25 . $S$ is the atomic orbital overlap matrix of the extended molecule. Equation (2) thus represents an exact method to incorporate the effects of $H_{\text {leads }}$ into transport.

We model electrostatic effects, including the potential imposed by the contacts as well as screening by the free ionic charges in the electrolyte solution surrounding the molecule, with a linearized Debye-Hückel theory ${ }^{29}$ In such a theory, Poisson's equation for the total electric potential $\Phi(\mathbf{r})$ becomes

$$
\nabla^{2} \Phi(\mathbf{r})=\frac{2 n \mathrm{e}^{2}}{k_{B} \mathrm{~T} \epsilon} \Phi(\mathbf{r})
$$

where $n=.1 \mathrm{M}$ is the concentration of the solution, $-\mathrm{e}$ is the charge of an electron, $\epsilon$ is the dielectric constant of water, $k_{B}$ is the Boltzmann constant, and $\mathrm{T}$ is room temperature 30

We solve Eq. (4) for parallel-plate boundary conditions, where the plates are taken to lie at the metalmolecule interfaces, and take $\Phi(\mathbf{r})$ constant within the metal nanoclusters themselves. In the electrostatic gauge for which the voltages of the left and right lead add to zero, the solution of Eq. (44) in the region containing the molecule is

$$
\Phi(z)=\frac{V}{2}\left[\cosh \left(\frac{z}{\lambda}\right)-\frac{\cosh \left(\frac{d}{\lambda}\right)+1}{\sinh \left(\frac{d}{\lambda}\right)} \sinh \left(\frac{z}{\lambda}\right)\right]
$$

where $z=0$ is one boundary surface, $z=d$ the other, $V$ the applied bias, and the Debye screening length is $\lambda=$ $\sqrt{k_{B} \mathrm{~T} \epsilon / 2 n \mathrm{e}^{2}}$. For the experimental systems of Refs. 2 and $3, \lambda=0.97 \mathrm{~nm}$; since this length is greater than $d / 2$ for all the metal-molecule-metal junctions studied here, the effect of screening in these systems is slight, and our results are robust against reasonable changes in the experimentally determined parameters T, $\epsilon, d$, and $n$. It should also be noted that, although Eq. (5) is written as an example in a particular gauge, the results of our calculations are gauge invariant.

We use a simple ansatz to include the electrostatic effect of $\Phi$ into $H_{m o l}: 31$

$$
\left(H_{m o l}\right)_{i j} \rightarrow\left(H_{m o l}\right)_{i j}-\mathrm{e} S_{i j} \frac{\Phi\left(\mathbf{r}_{i}\right)+\Phi\left(\mathbf{r}_{j}\right)}{2},
$$

where, $\mathbf{r}_{i}$ is the position of the $i^{\text {th }}$ atomic orbital. Thus, each diagonal element is gated in the usual way, and off-diagonal elements are modified according to the nonorthogonality of the basis states, as is typical in a Hückel theory. In addition, of course, the site energies of each lead and metallic nanocluster shift uniformly with their respective metallic contact's electrostatic potential. All the quantities in Eq. (2) thus become functions of the applied bias $V$, without the use of free parameters.

The Landauer formalism ${ }^{7}$ yields the conductance and current through the molecule. The probability for an 
TABLE I: Comparison of the conductances found in our mode ${ }^{8}$ (in units of $g_{0}$ ) for various bonding geometries with those of the STMBJ experiments. ${ }^{2,3}$ In the column headings, the first entry indicates the bonding geometry on the amine side of the molecule, the second the other. Only hollow bonding at both interfaces is compatible with experiment.

\begin{tabular}{|l|l|l|l|l|l|}
\hline molecule & experimental & hollow-hollow & hollow-top & top-hollow & top-top \\
\hline \hline cysteamine-Cys & $1.8 \times 10^{-4}$ & $5.4 \times 10^{-5}$ & $1.5 \times 10^{-6}$ & $9.6 \times 10^{-7}$ & $5.8 \times 10^{-9}$ \\
\hline cysteamine-Gly-Cys & $4.2 \times 10^{-6}$ & $4.3 \times 10^{-6}$ & $5.3 \times 10^{-8}$ & $3.7 \times 10^{-8}$ & $1.5 \times 10^{-9}$ \\
\hline Cys-Gly-Cys & $5.3 \times 10^{-6}$ & $4.2 \times 10^{-6}$ & $9.3 \times 10^{-8}$ & $1.0 \times 10^{-7}$ & $1.7 \times 10^{-9}$ \\
\hline cysteamine-Gly-Gly-Cys & $5.0 \times 10^{-7}$ & $4.1 \times 10^{-8}$ & $7.3 \times 10^{-10}$ & $1.9 \times 10^{-10}$ & $2.2 \times 10^{-11}$ \\
\hline
\end{tabular}

electron of energy $E$ to move from the left set of leads to the right is

$$
T(E, V)=\sum_{\substack{\alpha \in \text { left } \\ \beta \in \text { right }}} \operatorname{Tr}\left[\Gamma^{(\beta)}(E, V) G(E, V) \Gamma^{(\alpha)}(E, V) G^{\dagger}(E, V)\right]
$$

where

$$
\Gamma^{(\alpha)}(E, V) \equiv-2 \operatorname{Im}\left[\Sigma^{(\alpha)}(E, V)\right]
$$

is the broadening function for the term of $\Sigma$ from lead $\alpha$. The zero-bias conductance of the metal-moleculemetal junction is $g=g_{0} T\left(E_{F}, 0\right)$, with the conductance quantum $g_{0}=2 \mathrm{e}^{2} / h$ and the bulk gold Fermi energy $E_{F}$ evaluated for a 459-atom spherical gold nanocluster. The current is 7

$$
I(V)=\frac{\mathrm{e}}{h} \int_{-\infty}^{\infty} d E T(E, V)\left[f\left(E-\mu_{R}\right)-f\left(E-\mu_{L}\right)\right],
$$

where $f\left(E-\mu_{R, L}\right)$ is the Fermi function of the leads on the right (left) side of the extended molecule, and $\mu_{R, L}(V)$ is the corresponding electrochemical potential.

\section{RESULTS AND DISCUSSION}

\section{A. Conductance and Bonding Geometry}

We studied the conductance and current of each oligopeptide molecule measured experimentally in Refs. 2 and 3, varying several parameters of the experimental geometry in each case. These included the sulfur-to-sulfur distance as the molecule was stretched, the presence of additional molecules of the self-assembled monolayers, and bond geometry; only the last was found to have an impact on the observables of more than a factor of order unity. Figure 2 demonstrates this dependence, considering all four possibilities involving the two most likely bonding geometries of the sulfur: ${ }^{32}$ either directly to a single gold atom on top of the $\mathrm{Au}(111)$ substrate, or above a triangular hollow. Table 1 compares the conductances obtained from our mode ${ }^{8}$ with experiment.

The experimental data are consistent only with the model calculations for a hollow bonding geometry at both ends of each oligopeptide molecule. Since this
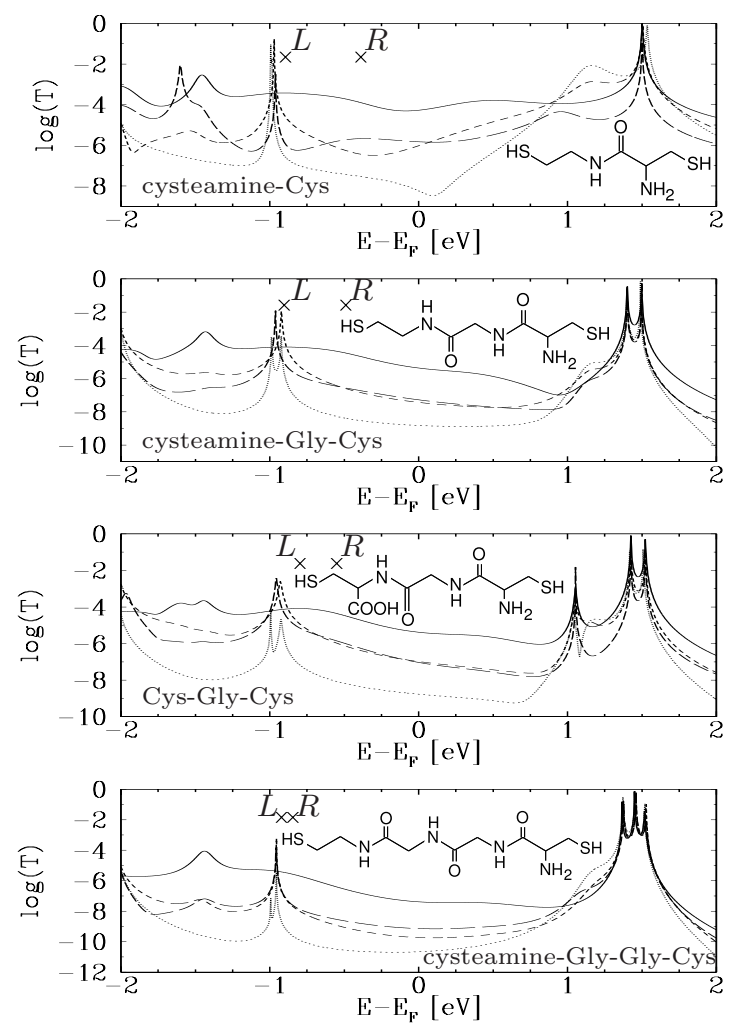

FIG. 2: Equilibrium transmission functions ${ }^{8} T(E, 0)$ for various bonding geometries for each of the four oligopeptide molecules studied experimentally in Refs. 2 and 3 . Solid lines indicate hollow-site bonding geometry on both sides. Dotted: top-site at both metal-molecule interfaces. Long dashes: hollow-site bonding for the sulfur on the amine-terminated side of the molecule and top-site bonding for the other. Short dashes: vice versa. Experimental conductances agree well with the solid curves in all cases. For the hollow-hollow geometry, the energies of the two molecular orbitals responsible for rectification are indicated with crosses. Inset: Structural formulae of the gas-phase molecules. When bonded to gold, the terminating hydrogen atoms are lost.

model is known to produce quantitatively correct conductance in other gold-thiol systems, $15,16,18,33$ we conclude that this is indeed the experimental geometry, and that the orders-of-magnitude smaller conductances associated with top-bound geometries and with molecules bound via monatomic gold chains ${ }^{34}$ form part of the low- 
conductance noise in the experimental histograms. ${ }^{2}$ In this way, the peaks of the conductance histograms of the STMBJ technique correspond to a specific geometry for the metal-molecule--metal junction, despite sulfur's ability to bond to a gold substrate in several geometries.

Further evidence that the single oligopeptide molecules of the experiments are bound to hollow substrate sites at each end is found in the behavior of conductance as a function of molecular stretching. Using DFT relaxation at progressively larger lead-lead distances, we find that internal degrees of freedom within the oligopeptide molecule itself accommodate only about one third of the total stretching induced by pulling the leads apart. The remaining stretching is allowed only by repositioning of the entire molecule between the two metal contacts, i.e. by changes of the Au-S-C bond angles and the dihedral angles involving both gold contacts and the molecule. This finding is consistent with the persistence lengths of polypeptide chains, typically several nanometers or more.

Indeed, the tetrahedral $s p^{3}$ bonding of the hollowbound geometry is much less flexible, and its symmetry more restrictive, than the linear $s p$ bond of the top-bound geometry. Therefore, when the hollow-hollow geometry is stretched, the DFT geometry optimizations only allow stretching of $.2 \AA$ or less (which affects $T(E)$ by $10 \%$ or less) before the molecular wire detaches from one electrode. By contrast, we find stretching distances as large as $1 \AA$ in top-bound geometries, and the conductance of unstretched top-bound geometries can be as much as 3-4 times larger than that of the fully stretched configurations reported in Table I as shown in Fig. 3. Since the experimental data ${ }^{2,3}$ exhibit no strong stretching dependence of conductance within a single plateau, we conclude that the experimental results correspond to the hollowhollow geometry, 35 in agreement with the conclusion reached above based on the magnitude of conductance. 36

Krüger et al. have suggested 34 that when a molecular wire thiol-bonded to gold contacts is stretched and eventually breaks, the cleavage does not occur at the gold-thiol interface, but instead that a gold atom, remaining attached to the thiol group, is pulled from the contact. During this process, the system passes through several intermediate, temporary configurations, including structures resembling the top-bound geometry discussed above. While a complete analysis of this geometric evolution and accompanying conductance changes is beyond the scope of the present work, we note that the results shown in Table Iindicate that the transition from hollowhollow to a top-bound geometry at either end is associated with a drop in conductance of more than an order of magnitude in each oligopeptide molecule. Thus, in a stretching experiment, when the top-bound geometry forms, the conductance plateau ends and the molecule appears to have simply separated from one or both contacts.

Furthermore, during stretching, temporary intermediate configurations such as those in Ref. 34 may form, which in general are less conducting than the original
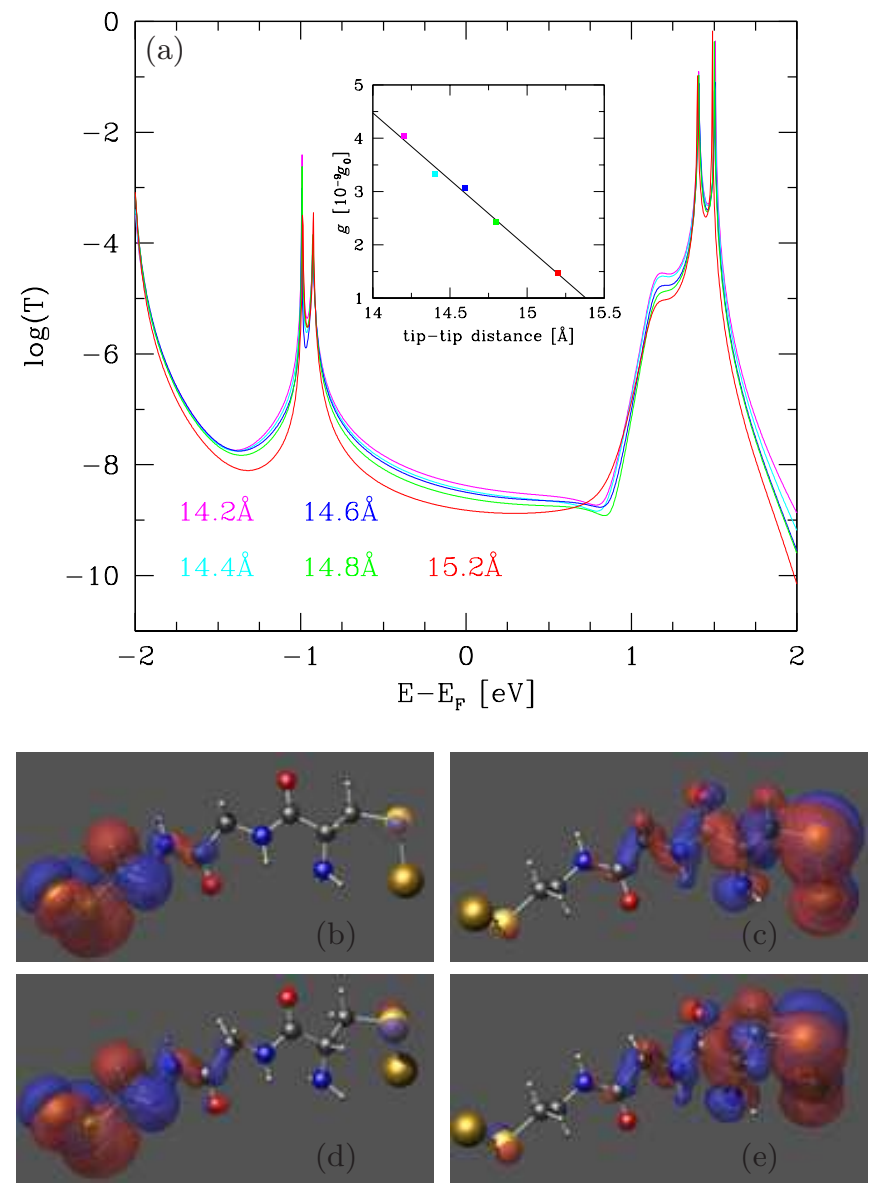

FIG. 3: (Color online) (a) Equilibrium transmission functions $T(E, 0)$ for cysteamine-Gly-Cys in the top-top configuration, for different distances between the two gold leads. Conductances of the metal-molecule-metal junction, shown in inset as a function of tip-tip distance, vary approximately linearly over a range of about $1 \AA$, beyond which no stable molecular structure bridges the two electrodes. Thus, the amount of stretching can affect conductance by a measurable amount, though not sufficiently to reconcile single-Au bonding geometries with the experimental results. For the hollow-hollow configuration, on the other hand, this range is much smaller, and so almost no variation in conductance is allowed. (b) and (c) show the $L$ and $R$ states, respectively, for the top-top simplified extended molecule with a tip-to-tip distance of $14.2 \AA$. (d) and (e) show the same for a tip-to-tip distance of $15.2 \AA$. Most of the stretching is accommodated by the bond angles at the gold-molecule interfaces, so that the principal change in (a) is peak widths.

configuration. One probable intermediate bonding geometry has the sulfur directly above a single gold atom embedded within the top atomic layer of the contact; when one end of the molecule is bound in such a way to the gold, and the other is in the hollow configuration, we find the molecular junction has a conductance of $1 / 4$ that of the hollow-hollow configuration. Indeed, weak features of lower conductance are seen in some of the ex- 
perimental conductance vs stretching curves of Ref. 2 . They are typically much shorter than the main plateau, and occur at its end, consistent with configurations that are only meta-stable and occasionally populated via the stretching mechanism.

To understand the transmission curves, it is helpful to think in terms of a smaller extended molecule which includes only the two to six gold atoms to which the sulfurs bond. Transmission states near $E_{F}$ correspond to gold-sulfur interfacial orbitals of this simplified extended molecule. Because there are two metal-molecule interfaces, these states typically occur in pairs with similar energies. When the two states are detuned from each other, they are largely localized at either end of the molecule; when in resonance, they mix into bonding and antibonding combinations (see Fig. (1). For a symmetric extended molecule at zero bias, the pairs are always in resonance; oligopeptides, however, are intrinsically asymmetric, so interfacial states can be detuned from each other and localized at either end, even in equilibrium.

Prominent peaks in the transmission spectra (Fig. 2) occur near the energies of molecular orbitals which bridge the left and right leads. Thus, the resonances, and consequent hybridization, of levels otherwise localized on opposite ends of the extended molecule play a dominant role in determining electron transport. For example, in all molecules and bonding configurations studied here, the two levels immediately below $E_{F}$ are paired interfacial states, which we call $R$ (localized at the gold-molecule interface near the amine terminus of the molecule) and $L$ (localized at the other interface). The narrow transmission peaks associated with their bonding and antibonding combinations are visible $1 \mathrm{eV}$ below $E_{F}$ in the double on-top (dotted) curves of Fig. 2, Because the overlap of the gold $6 s$ and sulfur $3 p$ orbital is strongly angle dependant, $\stackrel{37}{=}$ these peaks each become broadened when their respective bonding geometries are changed to hollow. Furthermore, the hollow-bonded versions of $R$ are more sensitive to the asymmetric features of the oligopeptide molecules, resulting in a significant detuning from $L$ (shown by the crosses in Fig. (2). The transmission peak associated with these two states is thus also severely broadened, becoming a smooth shoulder in $T(E, 0)$.

\section{B. Current Rectification}

The movement and change under bias of $R$ and $L$, depicted in Fig. 1] give rise to the rectification observed by Xiao et al. in Ref. 3. Positive bias raises (lowers) the electrochemical potential of atomic orbitals on the amine (non-amine) side of the molecule; consequently, under negative bias the energies of $R$ and $L$ move closer together. Near resonance, $R$ and $L$ hybridize into wholemolecule states, allowing significant charge transport.

Figure 4 shows the movement under bias of several generalized eigenenergies of the simplified extended molecule for hollow-hollow bonded cysteamine-Gly-Cys $\stackrel{8}{\underline{8}}$ All of

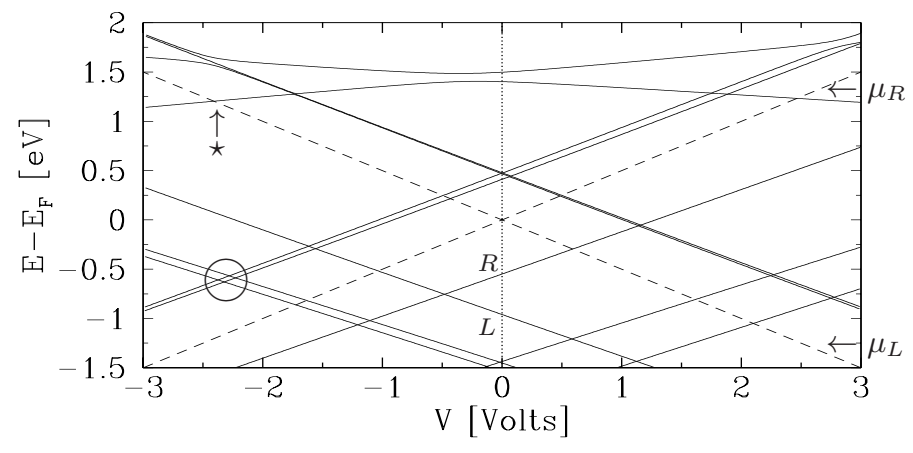

FIG. 4: Movement with applied bias of the generalized eigenvalues ${ }^{8}$ of the six-gold-atom simplified extended molecule for cysteamine-Gly-Cys in the hollow-hollow configuration. Some lines are doubled by degrees of freedom in the gold contacts; when more gold atoms are included, the molecular orbitals split further, eventually developing into the continuous density of states of an infinite system. Note that the apparent intersections are actually avoided crossings. In equilibrium, states $R$ and $L$ are detuned by $.4 \mathrm{eV}$ due to molecular asymmetry. They come into resonance only under negative bias, causing current rectification. Without these two states, the location of anticrossings, and thus the $I-V$ characteristic, would be largely symmetric. The dashed lines represent the electrochemical potentials of the right and left electrodes, $\mu_{R}$ and $\mu_{L}$. NDR is the results of increasing bias beyond the last anticrossing in a group; an example is circled.

the states in the region around $E_{F}$ occur in right/left pairs like $R$ and $L$, but only $R$ and $L$ are detuned from each other in equilibrium. Without $R$ and $L$, the locations of anticrossings are nearly symmetric about $V=0$. Rectification is a result of the splitting between these two states, itself a direct consequence of oligopeptide asymmetry.

Figure 5 gives the calculated current-voltage characteristic for each molecule, $\underline{\underline{8}}$ showing this rectification. While the resonance depicted in Fig. 1 is comparatively narrow and outside the energy window between $\mu_{L}$ and $\mu_{R}$, rectification extends beyond $2 \mathrm{~V}$ in the full systems due to the strong hollow-site broadening of $R$ and $L$. For the three molecules for which Xiao et al. made $I-V$ measurements (shown in inset), the calculated rectification is in excellent qualitative agreement with experimental data. We find a current ratio of about $150 \%$ for cysteamine-GlyCys and Cys-Gly-Cys, similar to Ref. 3. Cysteamine-GlyGly-Cys exhibits less rectification, both in our model and the experimental data: This is due to its much smaller detuning between $R$ and $L$ at zero bias.

\section{Negative Differential Resistance}

Negative differential resistance $(d I / d V<0)$ can result from applied bias destroying a resonance between transmitting interfacial states $\frac{38,39}{19}$ As the bias increases past an anticrossing in the metal-oligopeptide-metal systems, the two interfacial states are taken further apart in en- 

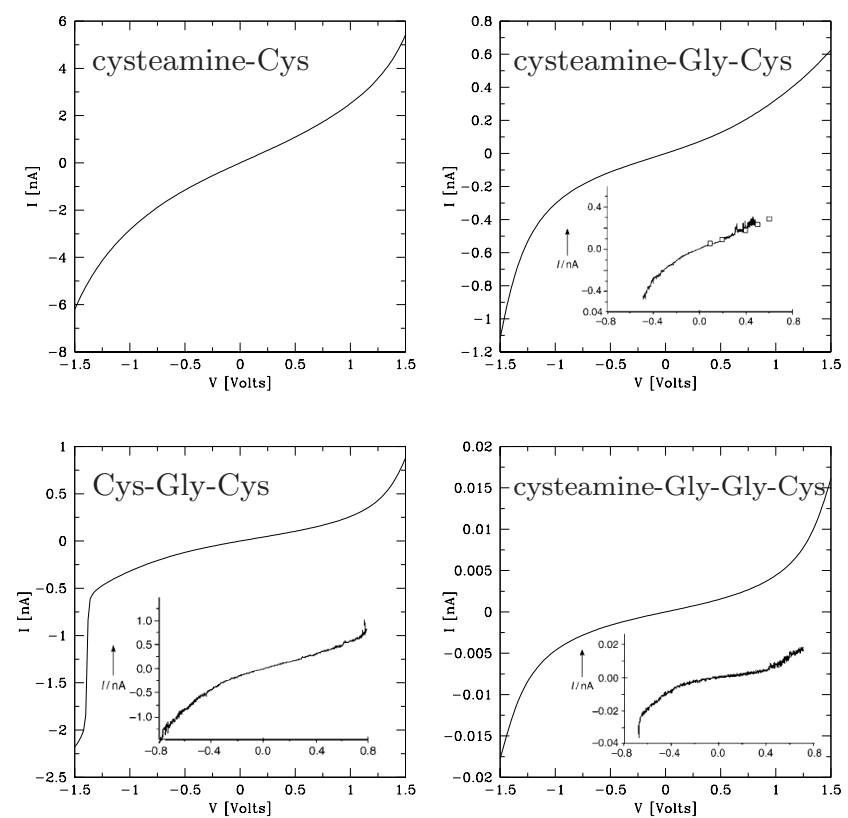

FIG. 5: Calculated current-voltage characteristics of four single-molecule oligopeptide devices $\stackrel{-8}{-}$ The scale and asymmetry of the current are comparable to those found in the experimental studies of Ref. 3, reproduced here in inset.

ergy. They therefore mix less well, and become localized at the individual metal-molecule interfaces. As the bias voltage increases, then, electron transport through these states "shuts off", creating the potential for NDR.

Such effects are masked while the current is still small: The dominant effect is the increasing current from a series of crossings and states entering the window of integration. Nevertheless, once the voltage is large enough that an entire neighborhood of interfacial resonances has been encompassed, further applied bias yields NDR. One such crossing is indicated by the circle in Fig. 4 its $I_{-}$ $V$ characteristic and relevant transmission properties are shown in Fig. 6. As $V$ becomes more negative, the peak in $T(E)$ associated with the nearly degenerate hybridized states diminishes. After the final current step, due to a mid-molecule orbital ( $\star$ in Fig. (4) entering the window of integration, NDR results. The bias voltages required to observe this important phenomenon are only moderately larger than those already achieved experimentally $\underline{\underline{3}}$

The magnitude of NDR is determined by the participating levels' electrostatic and quantum mechanical couplings to the leads. While the first is necessarily high for interfacial levels (hence the saturated slope of most lines in Fig. (4), the quantum mechanical coupling, or broadening, of an interfacial level depends mainly on the bond geometry at the metal-molecule interfaces. For hollow bonding, for example, interfacial states are very well broadened, as can be seen from the hollow-hollow (solid) curves of Fig. 2. On the other hand, if one or both of the interfaces is in the top-bound geometry, the related

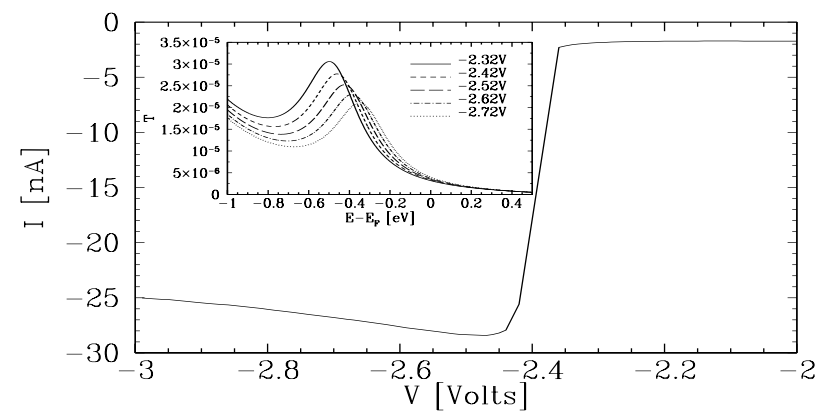

FIG. 6: $I-V$ characteristic for the hollow-hollow goldcysteamine-Gly-Cys-gold system ${ }^{8}$ in the medium-bias regime, showing NDR. Other oligopeptide molecules have qualitatively similar characteristics. (inset) Corresponding $T(E, V)$ curves. NDR corresponds to a gradual decrease in the transmission peak's strength as voltage becomes more negative, due to departure from a resonant crossing of interfacial states (the circle in Fig. 4).

\section{NDR is stronger.}

Like their rectifying properties, the NDR that we predict in oligopeptides is a direct result of the dominance of resonant crossings of interfacial states in charge transport. Since this is a property of all thiol- (i.e. cysteamine- or cysteine-) bonded oligopeptide molecules, both effects are robust, and observable in the entire class of such molecules. Because of the limitless customizability and simple fabrication of oligopeptides, the effects can be tailored to meet a wide variety of device needs.

\section{CONCLUSIONS}

In summary, we have developed a theoretical model which explains all of the experimental data on charge transport through oligopeptide molecules. We obtain quantitative agreement with experiment by using a combination of appropriate, standard theoretical models (DFT for the molecular geometry; the linearized DebyeHückel model for the potential profile, including the electrostatic screening effect of the electrolyte solution; and the extended Hückel model of Hoffman and coworkers 19.20 for the electronic structure) and careful consideration of the experimental systems. We use no fitting of any kind to any experimental data involving electrical conduction through molecules. Quantitative agreement without the use of fitting parameters allows us to extract detailed chemical and physical information from the experiments, such as the conclusion that both ends of the molecule are bound to hollow sites on the $\mathrm{Au}(111)$ surfaces.

We understand the observed current rectification of oligopeptides as due to a hybridization of interfacial states lying at either end of the molecule, naturally detuned from each other in equilibrium due to the intrinsic asymmetry of peptide chains. When bias is applied 
in one direction, these states are brought into resonance with each other, forming two whole-molecule states which allow a stronger current to flow; such a resonance does not occur when the molecule is biased in the opposite direction. Our calculated current rectifying ratios agree well with those measured in experiment, again without the use of fitting parameters.

Finally, our model predicts NDR will appear at moderately higher bias voltages than have been explored experimentally in Refs. 2 and 3 . This phenomenon is understood as the natural counterpart to the rectifying physics: As bias is increased past the anticrossing of the two interfacial states, the states once again become localized at the metal-molecule interfaces, and current flow through these channels decreases. Since NDR has many attendant device applications, and oligopeptides offer a unique combination of customizability and scalability, it is our hope that this work will inspire much further study of their electron transport properties.

\section{Acknowledgments}

We thank Nongjian Tao, Joshua Hihath, and Ross Hill for useful discussions. This work was supported by NSERC and the Canadian Institute for Advanced Research. Some numerical computations presented in this work were performed on WestGrid computing resources, which are funded in part by the Canada Foundation for Innovation, Alberta Innovation and Science, BC Advanced Education, and the participating research institutions. WestGrid equipment is provided by IBM, Hewlett Packard, and SGI.
1 R. I. Gilmanshin, In K. Sienicki, ed. Molecular Electronics and Molecular Electronic Devices, Vol. 2 (CRC Press, Boca Raton, 1993), pp. 1-78.

2 X. Xiao, B. Xu, and N. Tao, J. Am. Chem. Soc. 126, 5370 (2004).

3 X. Xiao, B. Xu, and N. Tao, Angew. Chem. Int. Ed. 43, 6148 (2004).

4 B. Xu and N. J. Tao, Science 301, 1221 (2003).

${ }^{5}$ B. Xu, X. Xiao, and N. J. Tao, J. Am. Chem. Soc. 125, $16164(2003)$.

${ }^{6}$ X. Xiao, B. Xu, and N. J. Tao, Nano Lett. 4, 267 (2004).

7 S. Datta, Electron Transport in Mesoscopic Systems, vol. 3 of Cambridge Studies in Semiconductor Physics and Microelectronic Engineering (Cambridge University Press, Cambridge, UK, 2003), ISBN 0521599431.

8 All of the numerical results presented in this article (with the exception of those in Fig. 3) are for the largest value of the spacing between the gold electrodes (for each molecule and bond geometry between the molecule and gold contacts) for which the $a b$ initio density functional theory geometry optimization converges to a geometry in which the molecule bridges the two leads. This is consistent with the experimental procedures of Refs. 2 3 4,5,6, in which the transport measurements were performed on stretched metal-molecule-metal junctions.

9 A. van der Ziel, Solid-State Electron. 26, 333 (1983).

10 E. R. Brown, J. R. Söderström, C. D. Parker, L. J. Mahoney, K. M. Molvar, and T. C. McGill, Appl. Phys. Lett. 58, 2291 (1991).

11 T. P. E. Broekaert, B. Brar, J. P. A. van der Wagt, A. C. Seabaugh, F. J. Morris, T. S. Moise, E. A. Beam, III, and G. A. Frazier, IEEE J. Solid-State Circuits 33, 1342 (1998).

12 R. H. Mathews, J. P. Sage, T. C. L. G. Sollner, S. D. Calawa, C.-L. Chen, L. J. Mahoney, P. A. Maki, and K. M. Molvar, Proc. IEEE 87, 596 (1999).

13 Gaussian 98, Revision A.11.3, M. J. Frisch et al..

14 Inclusion of such a large metallic nanocluster between leads and molecule allows for a quantitative treatment of peak broadenings, which are essential to the phenomena we dis- cuss in this work. We have verified that the clusters we use are large enough that increasing their size further does not significantly affect the results.

15 S. Datta, W. Tian, S. Hong, R. Reifenberger, J. I. Henderson, and C. P. Kubiak, Phys. Rev. Lett. 79, 2530 (1997).

16 E. G. Emberly and G. Kirczenow, Phys. Rev. B 64, 235412 (2001).

17 M. A. Reed, C. Zhou, C. J. Muller, T. P. Burgin, and J. M. Tour, Science 278, 252 (1997).

18 J. G. Kushmerick, D. B. Holt, J. C. Yang, J. Naciri, M. H. Moore, and R. Shashidhar, Phys. Rev. Lett. 89, 086802 (2002).

19 R. Hoffman, J. Chem. Phys. 39, 1397 (1963).

20 J. H. Ammeter, H.-B. Bürgi, J. C. Thibeault, and R. Hoffman, J. Am. Chem. Soc. 100, 3686 (1978).

21 J. Cerdá and F. Soria, Phys. Rev. B 61, 7965 (2000).

${ }^{22}$ G. Kirczenow, P. G. Piva, and R. A. Wolkow, Phys. Rev. B 72, 245306 (2005).

23 D. Kienle, J. I. Cerda, and A. W. Ghosh, J. Appl. Phys. 100, 043714 (2006).

24 The numerical implementation used was the YAeHMOP package (ver. 3.0), by G. A. Landrum and W. V. Glassey, with the default extended Hückel perscription for that program, given in Ref. 20

25 V. Mujica, M. Kemp, and M. A. Ratner, J. Chem. Phys. 101, 6856 (1994).

${ }^{26}$ K. Hansen, S. K. Nielsen, M. Brandbyge, E. Lægsgaarrd, I. Stensgaard, and F. Besenbacher, Appl. Phys. Lett. 77, 708 (2000).

27 H. Mehrez, A. Wlasenko, B. Larade, J. Taylor, P. Grütter, and H. Guo, Phys. Rev. B 65, 195419 (2002).

28 E. Emberly and G. Kirczenow, Phys. Rev. Lett. 81, 5205 (1998).

29 L. D. Landau and E. M. Lifshitz, Statistical Physics, vol. 5 of Course of Theoretical Physics (Pergamon, Bristol, Great Britain, 1958).

30 These parameters are chosen in accordance with the supplementary information to Ref. 2, freely available on the internet.

31 E. G. Emberly and G. Kirczenow, Chem. Phys. 281, 311 
(2002).

32 X. Li, J. He, J. Hihath, B. Xu, S. M. Lindsay, and N. Tao, J. Am. Chem. Soc. 128, 2135 (2006).

33 The method outlined in the present paper gives a conductance of $1.3 \times 10^{-4} g_{0}$ for a hollow-hollow goldoctanedithiol-gold junction, in good agreement with the experimental results of Ref. 32 .

34 D. Krüger, H. Fuchs, R. Rosseau, D. Marx, M. Parrinello, Phys. Rev. Lett. 89, 186402 (2002).

${ }^{35}$ Here, we assume that small changes in the top-bound geometry's Au-S-C bond angle are energetically favored over forming the monatomic chain-forming process of Ref. 34 . If not, both geometries would exhibit the unsloped conductance plateaus seen in experiment.

36 The greater length of stretching sometimes observed in the experiments is presumably due to motion within the larger experimental system, outside the scope of the present study.

37 The effect of bond geometry on molecular transmission has been well studied. See, for example, E. G. Emberly and G. Kirczenow, Phys. Rev. B 58, 10911 (1998); M. Di Ventra, S. T. Pantelides, and N. D. Lang, Phys. Rev. Lett. 84, 979 (2000); P. E. Kornilovitch and A. M. Bratkovsky, Phys. Rev. B 64, 195413 (2001); C. Toher, A. Filippetti, S. Sanvito, and K. Burke, Phys. Rev. Lett. 95, 146402 (2005).

38 H. Dalgleish and G. Kirczenow, Nano Lett. 6, 1274 (2006).

39 H. Dalgleish and G. Kirczenow, Phys. Rev. B 73, 245431 (2006) 